Research Article

\title{
The Phlorotannin-Rich Fraction of Ecklonia cava Extract Attenuated the Expressions of the Markers Related with Inflammation and Leptin Resistance in Adipose Tissue
}

\author{
Myeongjoo Son,, ${ }^{1,2}$ Seyeon Oh, ${ }^{2}$ Junwon Choi, ${ }^{1,2}$ Ji Tae Jang, ${ }^{3}$ Chang Hu Choi, ${ }^{4}$ \\ Kook Yang Park, ${ }^{4}$ Kuk Hui Son $\left(\mathbb{D},{ }^{4}\right.$ and Kyunghee Byun $\mathbb{1}^{1,2}$ \\ ${ }^{1}$ Department of Anatomy \& Cell Biology, Gachon University College of Medicine, Incheon 21936, Republic of Korea \\ ${ }^{2}$ Functional Cellular Networks Laboratory, College of Medicine, Department of Medicine, \\ Graduate School and Lee Gil Ya Cancer and Diabetes Institute, Gachon University, Incheon 21999, Republic of Korea \\ ${ }^{3}$ Aqua Green Technology Co., Ltd., Smart Bldg., Jeju Science Park, Jeju 63309, Republic of Korea \\ ${ }^{4}$ Department of Thoracic and Cardiovascular Surgery, Gachon University Gil Medical Center, Gachon University, \\ Incheon 21565, Republic of Korea
}

Correspondence should be addressed to Kuk Hui Son; dr632@gilhospital.com and Kyunghee Byun; khbyun1@gachon.ac.kr

Received 12 September 2019; Revised 26 December 2019; Accepted 13 January 2020; Published 7 March 2020

Academic Editor: Anna Hejmej

Copyright (c) 2020 Myeongjoo Son et al. This is an open access article distributed under the Creative Commons Attribution License, which permits unrestricted use, distribution, and reproduction in any medium, provided the original work is properly cited.

\begin{abstract}
Obesity is associated with systemic chronic inflammation, and it induces central leptin resistance which blocks the appetitesuppressing effect of leptin and leptin resistance in adipocytes. In the present study, we evaluated the effects of Ecklonia cava extract (ECE), which contained rich phlorotannins, on inflammation and leptin resistance in the adipose tissue of a diet-induced obese model. Effects of ECE on fat deposition, inflammation, M1/M2 macrophage, and T-cell infiltrations were investigated, and leptin resistance and SOCS3 were also measured in adipose tissue. Furthermore, ECE attenuated the expression of inflammationrelated receptors such as TLR4 and RAGE and leptin resistance by reducing SOCS3 expression, increasing expression of leptin receptor in adipose tissue, and increasing lipolysis. ECE showed antiadiposity and anti-inflammatory effects, attenuated leptin resistance, and increased lipolysis in the diet-induced obese model. This study shows that ECE is a suitable dietary supplement candidate for the prevention or treatment of obesity or obesity-associated diseases, especially inflammation-related diseases.
\end{abstract}

\section{Introduction}

Obesity leads to chronic inflammation and changes the secretion patterns of adipokines in adipose tissue. In obese fat tissue, hypertrophic adipocytes express and secrete high levels of proinflammatory cytokines, such as tumor necrosis factor $\alpha$ (TNF- $\alpha$ ), interleukin (IL)-6, IL-8, and monocyte chemoattractant protein-1 (MCP-1) $[1,2]$. These cytokines also promote and aggravate adipose tissue inflammation by recruiting immune cells, including macrophages and $\mathrm{T}$ cells $[2,3]$. Low-grade chronic inflammation in adipose tissue is attracting increasing attention because it is viewed to underlie obesity-related complications [4], such as diabetes, coronary heart diseases, hyperlipidemia, hypertension, and cancer [5].

Leptin is perhaps the most well-known adipokine and is produced by adipocytes. Leptin regulates food intake and energy expenditure by interacting with hypothalamic nuclei and plays a crucial role in the maintenance of body weight [6]. In fact, circulating leptin levels are proportional to body fat, but in diet-induced obesity, these increased levels fail to prevent weight gain. This unresponsiveness to endogenous (or exogenous) leptin is referred to as "leptin resistance" $[7,8]$. Leptin acts by binding to functional long isoform leptin receptor (ObR) in the central nervous system (CNS), especially in the hypothalamus, and ObR is known to 
activate Janus-activating kinase 2-signal transducer/activator of transcription 3 (JAK2/STAT3) and phosphatidylinositol 3-kinase (PI3K) signaling pathways $[9,10]$. The ability of leptin in activating STAT3 and PI3K is diminished in diet-induced obese mice [10-12] and leptin increased expression of suppressor of cytokine signaling-3 (SOCS3) which is a main inducer of leptin resistance in the CNS. Furthermore, SOCS3 acts as a potent negative regulator of the JAK/STAT pathway and thus constitutes a negative feedback loop $[13,14]$. SOCS3 is also involved in the onset of leptin resistance at the central and peripheral levels [15]. Obesity promotes SOCS3 expression in the hypothalamus $[14,16]$, adipose tissue $[16,17]$, liver $[16,18]$, and skeletal muscle $[16,19]$, and SOCS3 might also upregulate the expressions of adipocyte inflammatory cytokines like IL-6, TNF- $\alpha$, and MCP-1 [20]. These results indicate that leptin resistance in adipocytes might influence adipocyte inflammation.

Ecklonia cava (E. cava) is a species of brown alga abundant in the neritic regions of Korea and Japan [21, 22] and has attracted research because of the medicinal effects of its components, which include carotenoids, fucoidans, and phlorotannins [21-26], and its anti-inflammatory, antioxidative, antidiabetic [27, 28], and antiobesity effects [21]. It has been reported that the E. cava extract (ECE) reduces body weight gain, body fat, and hyperglycemia, improves glucose tolerance due to its anti-inflammatory effects, and enhances lipid metabolism in a high-fat diet-induced (HFDinduced) animal model of obesity [21]. Although its antiinflammatory effects are well-established, little is known of its effects on inflammation and leptin resistance in adipose tissue relatively.

Garcinia cambogia is a tropical edible fruit of the family Clusiaceae and is grown in south East Asia, southern India, and Africa $[8,29]$. The dried fruit rinds of Garcinia cambogia contain $20-30 \%$ by weight of (-) hydroxycitric acid (HCA) [9], which is a competitive inhibitor of ATP-citrate lyase, a key enzyme involved in fatty acid and cholesterol biosynthesis $[8,30]$. Many studies have investigated the antiobesity effect of the Garcinia cambogia extract (GCE). For example, supplementation of HFD with GCE significantly reduced body weight gain, glucose intolerance, and plasma leptin and TNF- $\alpha$ levels in the obese mouse model [8]. In the present study, we evaluate the effects of ECE on inflammation and leptin resistance in adipose tissue in a HFD-induced obese animal model and compared its effects with those of GCE.

\section{Materials and Methods}

2.1. Preparations of ECE and GCE. The ECE was manufactured as follows. E. cava collected near the Jeju coast was washed thoroughly and air dried for $48 \mathrm{hr}$ at room temperature. The E. cava was extracted and finely grounded using $50 \%$ aqueous ethanol for $12 \mathrm{hr}$ at $85^{\circ} \mathrm{C}$. The E. cava extract was filtered, concentrated, and sterilized (40 to $60 \mathrm{~min}$ at $85^{\circ} \mathrm{C}$ ). Finally, a dry powder from the E. cava extract was produced by spray-drying. Phlorotannins of the prepared ECE was validated using HPLC (Figure S1). GCE was obtained from ESFood (Lot. 0831; Gunpo, Republic of
Korea), and the GCE containing 60\% HCA was used as a positive control.

2.2. Animal Study Design. Male mice C57BL/6N (7 weeks old) were purchased from Orient Bio (Republic of Korea) and maintained under controlled conditions (temperature is $23^{\circ} \mathrm{C}$ and $50 \%$ humidity under $12 \mathrm{hr}$ dark/light cycle). After seven days of acclimatization, mice were randomly divided into four groups in which (1) mice were fed a regular chow diet for 4 weeks and then orally administered $0.9 \%$ normal saline for 4 weeks (the NFD group) and (2) mice were fed a $45 \%$ high-fat diet (HFD; Research Diet Inc., USA) for 4 weeks and then orally coadministered $0.9 \%$ normal saline (the HFD/saline group), ECE (70 mg/kg/day; the HFD/ECE group), or GCE (500 mg/kg/day; the HFD/GCE group) for 4 weeks. After oral administration, visceral fat samples were collected from retroperitoneal adipose tissue in accordance with the ethical principles issued by the Institutional Animal Care and Use Committee of Gachon University (approval number: LCDI-2017-0034). Body weights and food intakes were measured weekly, and body masses of fat and lean mice were measured using the $1 \mathrm{Hminispec}$ system (Bruker Optik, LF90II; Germany).

\subsection{RNA Extraction and Quantitative Real-Time} Polymerase Chain Reaction ( $q R T-P C R$ ). The visceral fat tissues $(200 \mathrm{mg})$ were homogenized by using gentleMACS $M$ tubes (MiltenyiBiotec, Germany) in $500 \mu \mathrm{l}$ of RNiso (TAKARA, Japan). Homogenates were then added to $0.1 \mathrm{ml}$ of chloroform, mixed, and centrifuged at $12,000 \times \mathrm{g}$ for $15 \mathrm{~min}$ at $4^{\circ} \mathrm{C}$. Aqueous phases were collected, placed in cleaned tubes, mixed with $0.25 \mathrm{ml}$ of isopropanol, and centrifuged using the same conditions. Isolated RNA samples were then washed with $0.5 \mathrm{ml} 70 \%$ ethanol and dissolved in $30 \mu \mathrm{l}$ of diethyl pyrocarbonate- (DEPC-) treated water. For qRT-PCR (quantitative real-time polymerase chain reaction), cDNA was synthesized from $1 \mu \mathrm{g}$ of total RNA using a Prime Script 1st strand cDNA Synthesis Kit (TAKARA, Japan). Synthesized cDNA was used as a template and qRT-PCR was performed using the CFX 384 Touch TM Real-Time PCR detection system. Reaction efficiencies and threshold cycle numbers were determined using CFX Manager TM Software. Primer sequences for target genes are detailed in Table S1.

2.4. Paraffin Block Preparation. Visceral fat was harvested from retroperitoneal visceral fat. The fat tissues were fixed in $4 \%$ paraformaldehyde (Biosesang, Republic of Korea) overnight at $4^{\circ} \mathrm{C}$ and placed in an automatic dehydration machine (Leica, UK). Tissues were dehydrated in a series of steps, that is, with $90 \%$ ethanol 3 times for $1 \mathrm{hr}$ followed by $100 \%$ ethanol 2 times for $2 \mathrm{hr}$ and then cleared with $100 \%$ xylene 3 times for $1.5 \mathrm{hr}$ and embedded in paraffin.

2.5. Histologic Staining and Quantification. For visceral fat size measurements, visceral fat paraffin tissues were sectioned at $7 \mu \mathrm{m}$ and stained with hematoxylin (DAKO, Japan) 
and eosin (Sigma-Aldrich, USA). The stained images were obtained using an Axio Imager Z1 upright microscopy system (Carl Zeiss, Germany). Adipocyte areas were determined from the cross-sectional areas of adipocyte cell membranes using Image J software (NIH, USA).

2.6. Immunostaining. Visceral fat tissue sections were incubated with anti-TLR4 (dilution rate $1: 100$ ), anti-RAGE (dilution rate $1: 100$ ), anti-SOCS3 (dilution rate $1: 100$ ), and anti-ObR (dilution rate $1: 100$ ) antibodies for 1 day at $4^{\circ} \mathrm{C}$, rinsed twice with phosphate-buffered saline (PBS), incubated with fluorescent conjugated secondary antibodies for $1 \mathrm{hr}$ in blocking solution, and rinsed twice with PBS. To detect nuclei of fat tissue, DAPI (Sigma-Aldrich, USA) was used for $3 \mathrm{~min}$. The fluorescent images were detected with confocal microscopy (LSM 710; Carl Zeiss, Germany), and quantification of the intensity of the fluorescent was detected using ZEN software 2012 version (Carl Zeiss, Germany).

2.7. ELISA for Serum Leptin and Adiponectin. Serum samples were obtained by incubating collected blood at room temperature for $20 \mathrm{~min}$ and centrifuging at $2,000 \times \mathrm{g}$ for $10 \mathrm{~min}$ at $4^{\circ} \mathrm{C}$. Absolute concentrations of adipokines like leptin (Invitrogen, USA) and adiponectin (Abcam, UK) in serum were determined using ELISA kits. Absorbances were measured at $450 \mathrm{~nm}$ using an ELISA plate reader (Molecular Devices, USA).

2.8. Statistical Analysis. The Kruskal-Wallis test and the Mann-Whitney $U$ test as a post hoc test were used to determine the significances of differences among the NFD/ saline, HFD/saline, HFD/ECE, and HFD/GCE groups. Results are presented as mean $\pm S D$, and statistical significance was accepted for $p$ values $<0.05$. The analysis was performed using SPSS version 22 (IBM Corporation, USA).

\section{Results and Discussion}

3.1. Attenuating Effects of E. cava Extract on HFD-Induced Adiposity, Increased Food Intake, and Adipocyte Size. ECE attenuated HFD-induced body weight and fat mass gain more than GCE. Animals in the HFD/saline group gained significantly more weight than animals in the NFD/ saline group (Figure 1(a)), and animals in the HFD/ECE group gained significantly less weight than animals in the $\mathrm{HFD} /$ saline group. However, weight gain in the HFD/GCE group was not significant different from that in the HFD/ saline group. Fat mass was significantly greater in the HFD/ saline group than in the NFD/saline group. The fat mass and lean mass of the HFD/ECE group were significantly decreased than in the $\mathrm{HFD} /$ saline group, and they were significantly different in the HFD/ECE and HFD/GCE groups (Figures 1(b) and 1(c)). The lean mass was decreased significantly in the HFD/ECE group than that in the $\mathrm{HFD} /$ saline group, but the decrease was not significant in the HFD/GCE group than in the HFD/saline group (Figure 1(c)). In addition, ECE decreased food intake more than GCE, and food intake was higher in the HFD/saline group than in the NFD/saline group but lower in the HFD/ ECE group than in the HFD/saline group. However, food intakes in the HFD/GCE and HFD/saline groups were nonsignificantly different (Figure 1(d)). Furthermore, the mean white adipocyte sizes in visceral fat tissues in the $\mathrm{HFD} /$ saline, HFD/ECE, and HFD/GCE groups were greater than in the NFD/saline group and were significantly smaller in the HFD/ECE group than in the HFD/saline group (Figure 1(e)).

3.2. Effects of E. cava Extract on Activated Macrophage Infiltration, M1 Polarization, and T-Cell Infiltration in Adipose Tissue. The expression of CD11b (an activated macrophage marker) [31] mRNA in visceral fat was significantly higher in the $\mathrm{HFD} /$ saline group than in the NFD/saline group and significantly lower in the HFD/ECE and HFD/GCE groups than in the HFD/saline group. The CD11b expression was significantly lower in the HFD/ECE group than in the HFD/ GCE group (Figure 2(a)). CD80 expression (a M1 macrophage marker) $[32,33]$ was significantly lower in the HFD/ ECE and HFD/GCE groups than in the HFD/saline group. The CD80 expression in HFD/ECE was significantly lower than that in the HFD/GCE group. The CD206 (marker of M2) $[32,33]$ was significantly higher in the HFD/ECE group than in the HFD/saline group. The total number of macrophages in adipose tissue, which were classified as M1 (classically activated) and M2 phenotypes (alternatively activated) [2, 34], prominently increases with obesity. The M1 type prominently expresses inducible nitric oxide synthase (iNOS), TNF- $\alpha$, IL- $1 \beta$, and CD11c on cell surfaces, whereas the M2 type topically expresses arginase, IL-10, and Ym-1 [2]. It has been established that obesity induces the polarization of macrophages in fat tissues from the M2 to the M1 phenotype [35], and it has been reported that M1 macrophages are the major cause of adipose tissue inflammation and insulin resistance in obesity [2]. In the present study, ECE attenuated HFD-induced macrophage infiltration into fat tissues and M1 polarization more effectively than GCE (Figures 2(b) and 2(c)). The number of Th1, a subtype of CD4, and CD8 T cells is elevated in adipose tissues of obese subjects [2, 36-38]. The Th1 cell and CD $8 \mathrm{~T}$ cell are also major interferon gamma- (IFN $\gamma$-) expressing cells that accumulate in obese people [38]. IFN $\gamma$ stimulates the expression of chemokines and proinflammatory cytokines in adipocytes as well as the M1 polarization of macrophages [2]. In visceral fat tissue, the expressions of CD8a and CD4 mRNAs in T cells were significantly higher in the $\mathrm{HFD} /$ saline group than in the NFD/saline group, and these expressions were significantly lower in the HFD/ECE group than in the HFD/saline group. ECE attenuated HFDinduced T-cell expression significantly higher than GCE (Figures 2(d) and 2(e)). Like visceral fat tissues, the ECE and pyrogallol-phloroglucinol-6,6-bieckol (PPB), a representative phlorotannin from ECE, attenuate endothelial cell dysfunction by controlling perivascular fat tissue dysfunction through decreasing inflammation and ER stress and modulating brown adipocyte function [39]. 


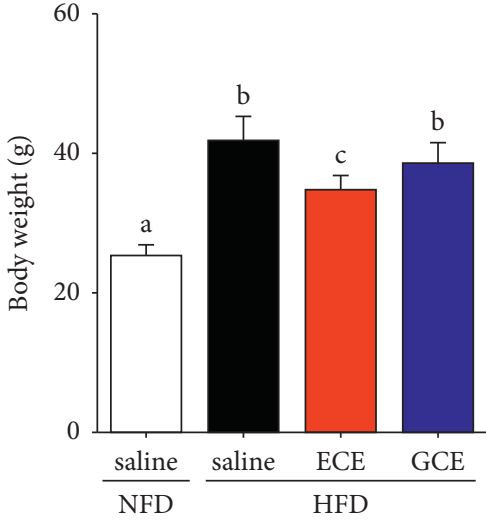

(a)

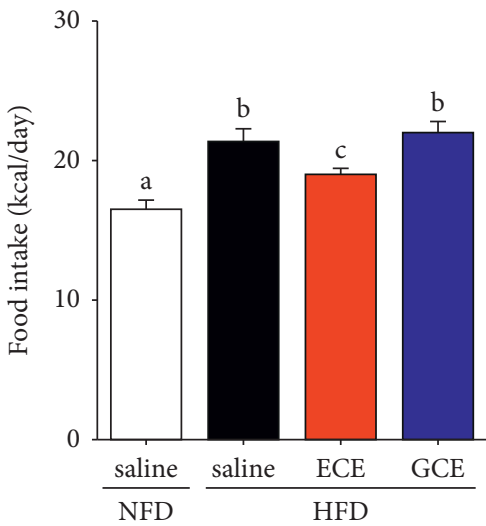

(d)

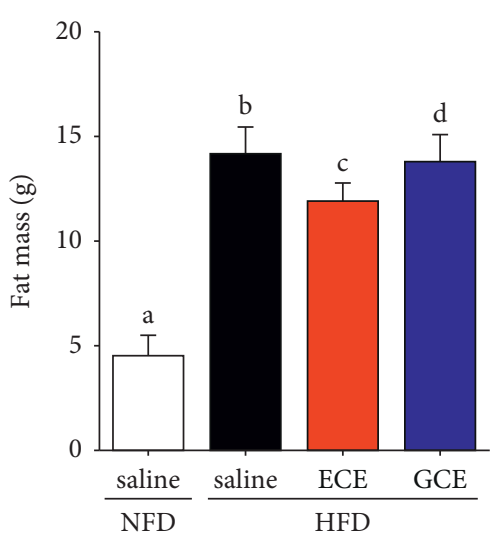

(b)

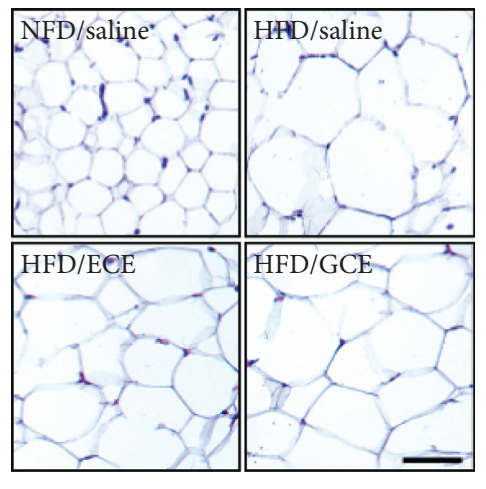

(e)

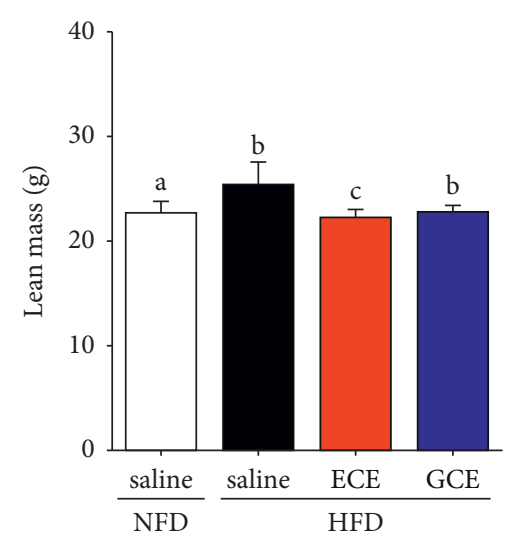

(c)

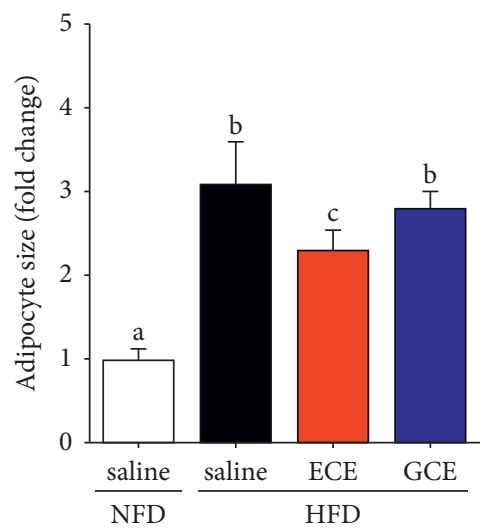

(f)

FIGURE 1: Effects of E. cava extract on body weight, adiposity, food intake, and adipocyte sizes in high-fat diet- (HFD-) induced obese mice. Mice fed with standard normal fat diet (NFD; open bar) or HFD (HFD; black bar) for 4 weeks and then $0.9 \%$ saline (NFD/saline group and HFD/saline group), $70 \mathrm{mg} / \mathrm{kg} /$ day E. cava extract (HFD/ECE group; red bar) or $500 \mathrm{mg} / \mathrm{kg} /$ day Garcinia cambogia extract (HFD/GCE group; blue bar) orally administrated for the next 4 weeks. After 8 weeks of administration, (a) body weight, (b) fat mass, (c) lean mass, and (d) food intake were measured. (e) Hematoxylin and eosin (H\&E) stained images showing adipocytes in visceral fat and (f) a graph showing average visceral adipocyte size in representative H\&E stained images. Adipocyte size was expressed as folds of that of NFD/saline group. Scale bar $=200 \mu \mathrm{m}$. The same letters represent no significant difference $(p<0.05)$.

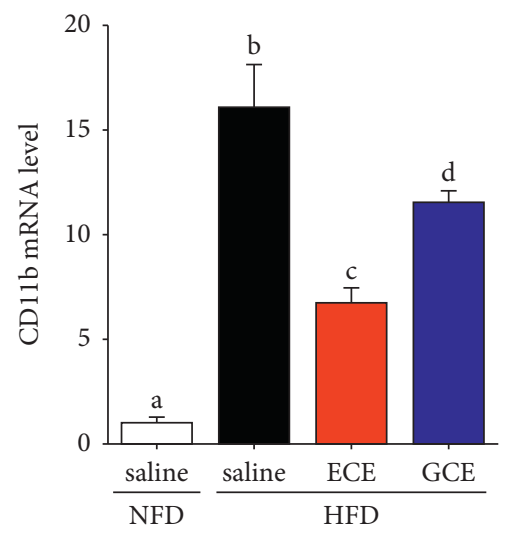

(a)

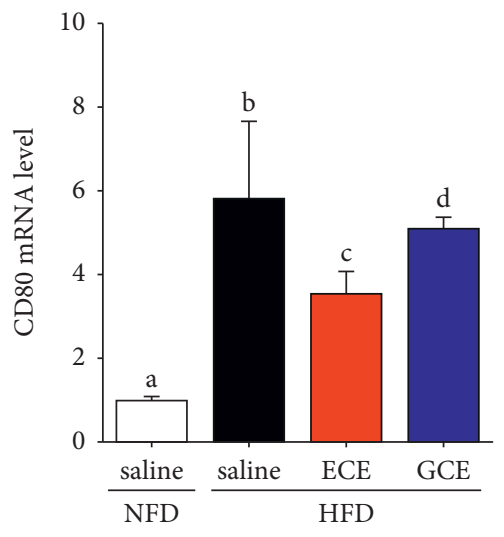

(b)

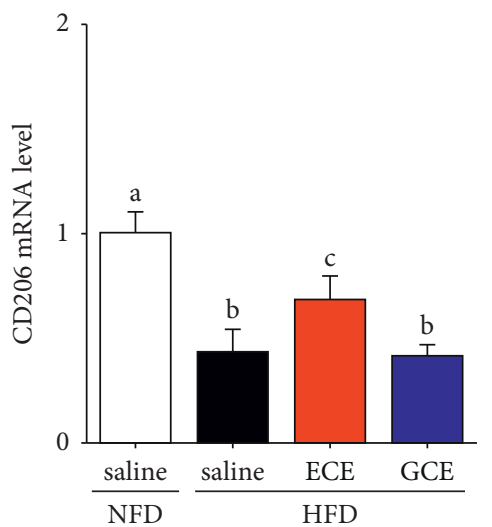

(c)

Figure 2: Continued. 


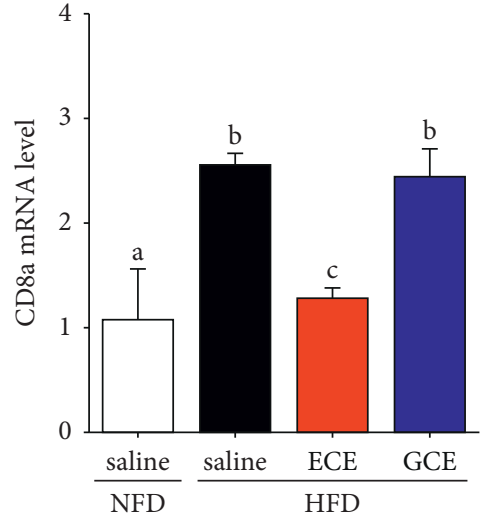

(d)

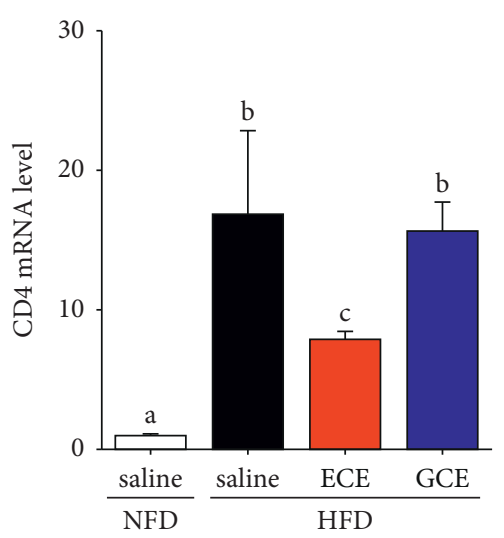

(e)

Figure 2: Effects of E. cava extract on macrophages and T-cell infiltration in visceral fat of high-fat diet- (HFD-) induced obese mice. (a) CD11b as a marker of total macrophage; (b) CD80 as a marker of M1 macrophage; and (c) CD206 as a marker of M2 macrophage expression mRNA levels were measured by qRT-PCR in visceral fat of standard normal fat diet with $0.9 \%$ saline (NFD/saline; open bar), HFD with $0.9 \%$ saline (HFD/saline; black bar), HFD with E. cava extract (HFD/ECE; red bar), and HFD with Garcinia cambogia extract (HFD/GCE; blue bar) groups. (d, e) CD8a and CD4 as T-cell expression levels were determined by qRT-PCR in visceral fat of the same groups. All mRNA levels expressed relative level and normalized $\beta$-actin of the NFD/saline group. The same letters represent no significant difference $(p<0.05)$.

3.3. Effects of E. cava Extract on Inflammatory Cytokine Production in Fat Tissue. Obesity-induced increases in M1 macrophage infiltration [40], T-cell numbers, and hypertrophic adipocyte numbers [2] have been reported to enhance proinflammatory response in adipose tissue and to increase secretions of inflammatory cytokines (e.g., TNF- $\alpha$ and IL-6) and decrease secretion of anti-inflammatory cytokine (e.g., IL-10 or TGF- $\beta$ ) [40-42]. We observed that the expressions of IL- 6 and TNF- $\alpha$ mRNA were significantly higher in the HFD/saline group than in the NFD/saline group. The inflammatory cytokines were decreased in both of the HFD/ECE and HFD/GCE groups, and the decreasing effect of HFD/ECE group was more prominent than that of the HFD/GCE group (Figures 3(a) and 3(b)). In addition, the expressions of IL-10 and TGF- $\beta$ mRNA were significantly lower in the HFD/saline group than in the NFD/saline group. These levels were increased in the HFD/ECE group, and these levels in the HFD/ECE group were greater than those in the HFD/GCE group (Figures 3(c) and 3(d)).

\subsection{Effects of E. cava Extract on TLR4, RAGE, and NF- $\kappa B$} Protein Expressions in Adipose Tissue. The productions of inflammatory cytokines, such as TNF- $\alpha$, IL- 6 , and IL- $1 \beta$, are induced by TLR4 signaling $[43,44]$. In one in vitro study, it was shown that ECE acts as a negative regulator of inflammation by suppressing TLR4 signaling and the secretions of TNF- $\alpha$, IL- 6 , IL- $1 \beta$, and IL-10 by lipopolysaccharidestimulated macrophages [43]. In our study, ECE attenuated TLR4 expression in visceral adipose tissue (Figures 4(a) and 4(b)). Advanced glycation end products (AGEs), members of the proinflammatory S100/calgranulin family, and high motility group box 1 protein are the ligands which bind to the receptor for advanced glycation end products (RAGE) $[45,46]$. Inflammation-related signaling cascades involving nuclear factor (NF)- $\kappa \mathrm{B}$ (Figures $4(\mathrm{e})$ and Figure S2), extracellular signal-regulated kinase (ERK) 1/2, mitogen-activated protein kinases (p38 MAPK), c-Jun N-terminal kinases (JNK), protein kinase C (PKC), Rac/Cdc42, or TIRdomain containing adapter protein (TIRAP) and MyD88 (adapter proteins for TLR2 and 4) are activated by binding RAGE and its ligands $[45,46]$. These observations regarding the potential regulatory role of RAGE in inflammation suggest that RAGE might importantly contribute to inflammation and to the obesity-associated dysregulation of adipokines [47]. Several studies have shown that RAGE is related to obesity. Especially, the adipocytes of obese human show higher RAGE expression than those of the lean human [47] and accumulate RAGE ligands as well in the adipose tissues of HFD and obesity models [48-50]. In our study, ECE reduced TLR4, RAGE, and NF- $\kappa \mathrm{B}$ expressions in the adipose tissues of HFD-fed mice (Figures 4(c) and 4(d)).

\subsection{Effects of E. cava Extract on Serum Leptin and Adiponectin} Levels and on Leptin Resistance in Adipose Tissue. Adipokine production is altered as adipose tissue mass increases. Most notably, adiponectin production drops and results in reduced glucose uptake and anti-inflammatory regulation of the local environment [22] and adipocytes start producing more leptin [28]. In our study, HFD caused decrease in adiponectin levels, and ECE attenuated this decrease more so than GCE. Also, HFD-induced leptin increases were reduced more by ECE than GCE (Figures 5(a) and 5(b)). Wang et al. proposed that white adipose tissue develops local leptin resistance, which may partially explain why circulating high concentrations of leptin in obese animals show little effect on white adipose tissue mass as well as the development of central leptin resistance in the central nerve system (CNS) [51, 52]. They also reported that activation of STAT3 was diminished in adipose tissue from leptin-resistant diet-induced obese rats under basal and 


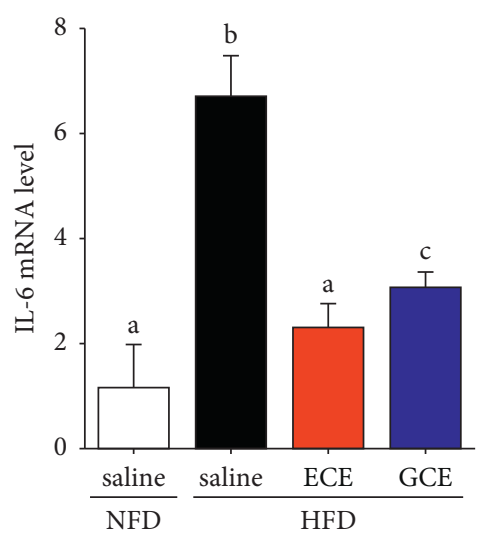

(a)

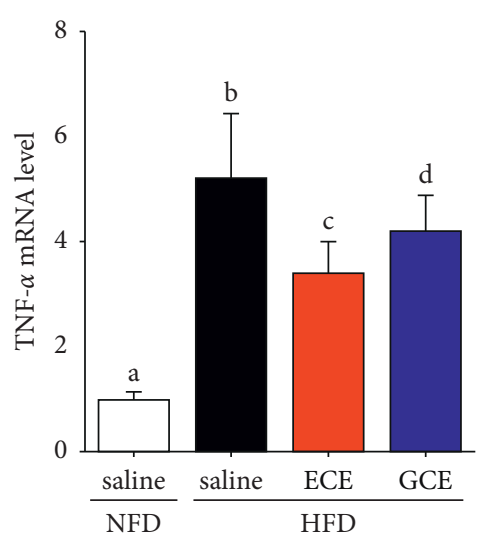

(b)

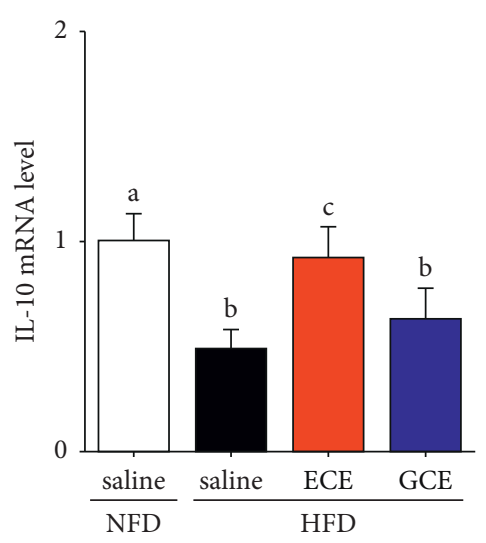

(c)

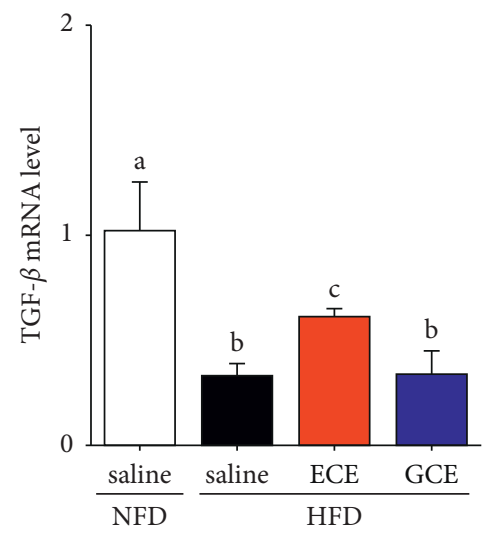

(d)

FIGURE 3: Effects of E. cava extract on inflammatory cytokines expression in visceral fat on high-fat diet- (HFD-) induced obese mice. (a, b) IL-6 and TNF- $\alpha$ as M1 macrophage-related inflammatory cytokines and (c, d) IL-10 and TGF- $\beta$ as M2 macrophage-related cytokines in visceral fat of standard normal fat diet with $0.9 \%$ saline (NFD/saline; open bar), HFD with $0.9 \%$ saline (HFD/saline; black bar), HFD with $E$. cava extract (HFD/ECE; red bar), and HFD with Garcinia cambogia extract (HFD/GCE; blue bar) group. All expression levels were determined by qRT-PCR, and all mRNA levels expressed relative level and normalized $\beta$-actin of the NFD/saline group. The same letters represent no significant difference $(p<0.05)$.

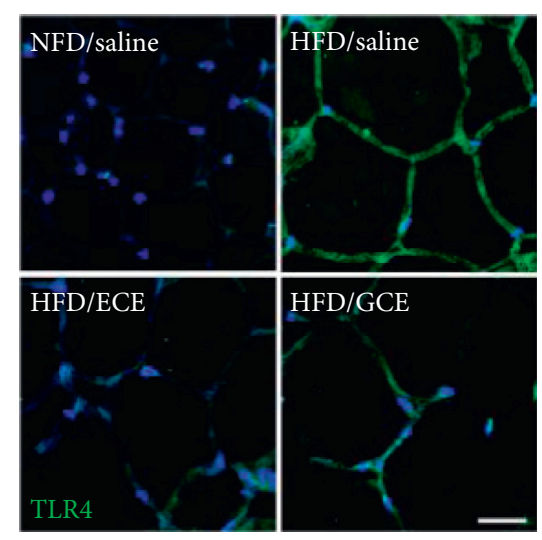

(a)

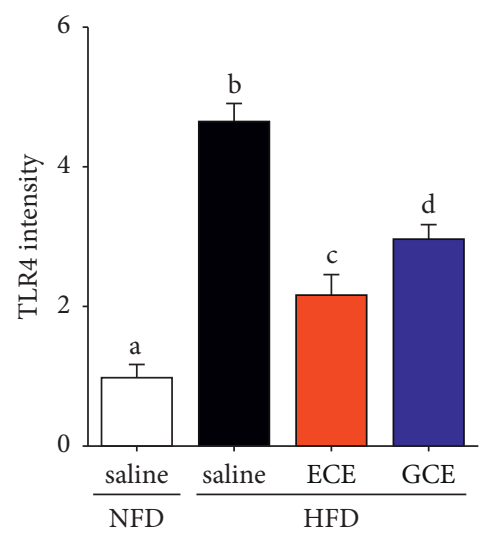

(b)

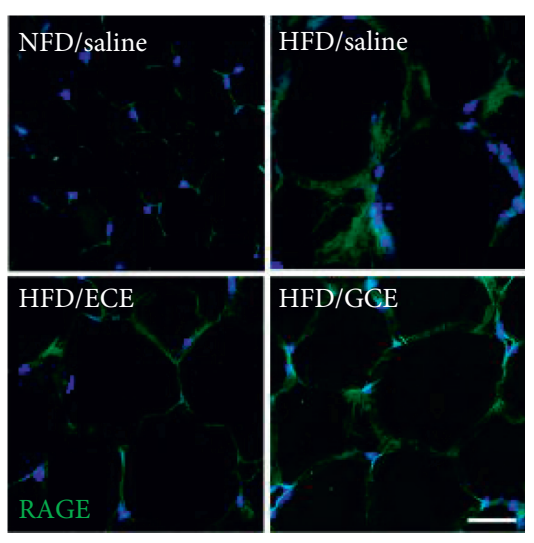

(c)

Figure 4: Continued. 


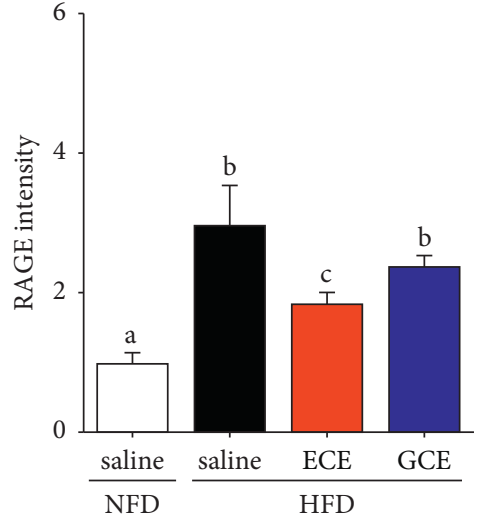

(d)

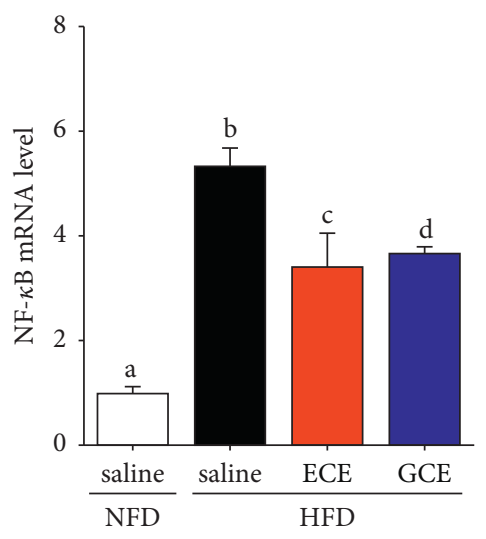

(e)

FiguRE 4: Effects of E. cava extract on TLR4, RAGE, and NF- $\kappa$ B expressions in visceral fat of high-fat diet- (HFD-) induced obese mice. To validate expression of inflammation-related receptors in visceral fat of standard normal fat diet with $0.9 \%$ saline (NFD/saline; open bar), HFD with $0.9 \%$ saline (HFD/saline; black bar), HFD with E. cava extract (HFD/ECE; red bar), and HFD with Garcinia cambogia extract (HFD/GCE; blue bar) group, tissue section slides were prepared. (a, c) The confocal microscopic images show toll-like receptor 4 (TLR4; (a) green), the receptor of AGE (RAGE; (c) green) expressions were assessed by immunostaining, and nuclei were stained DAPI (blue). (b, d) Quantitative green fluorescence intensity graphs show folds of those of the NFD/saline group, and these graphs show average expression levels in representative images. (e) NF- $\kappa \mathrm{B}$ expressions were assessed by $\mathrm{qRT}$-PCR. Scale bar $=50 \mu \mathrm{m}$. The same letters represent no significant difference $(p<0.05)$.

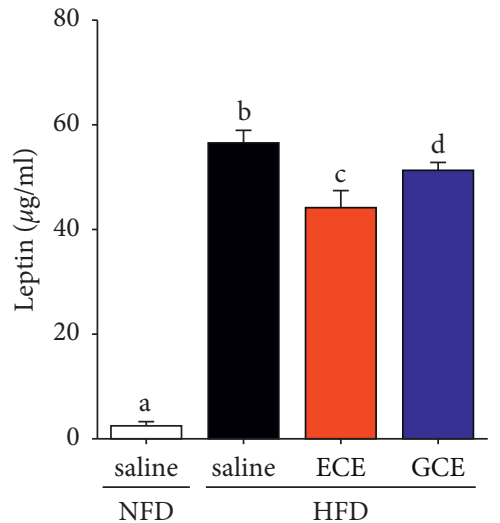

(a)

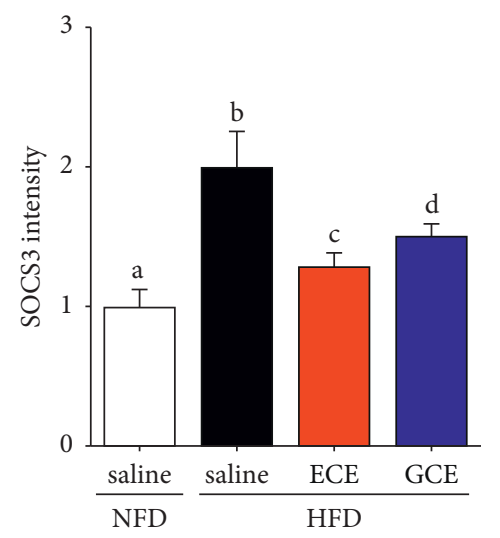

(d)

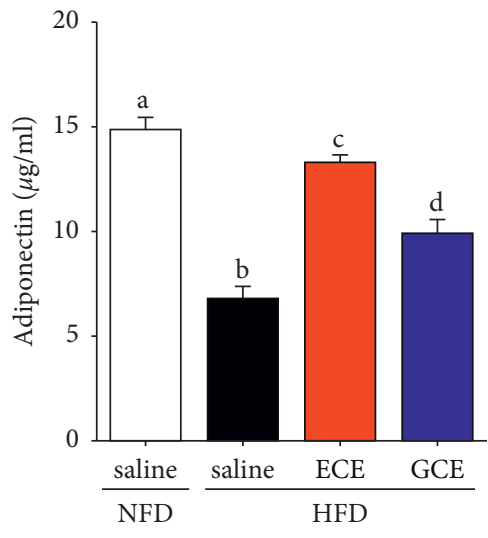

(b)

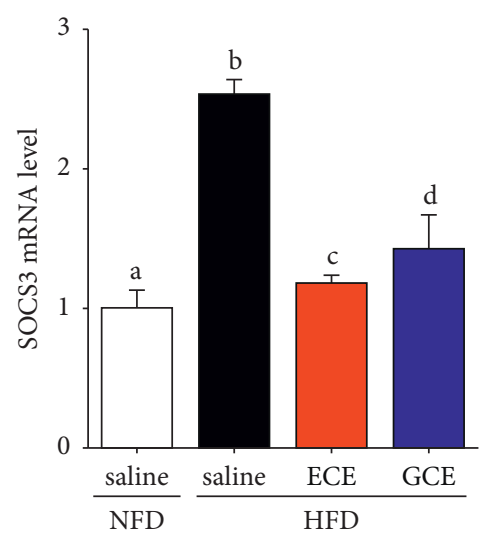

(e)

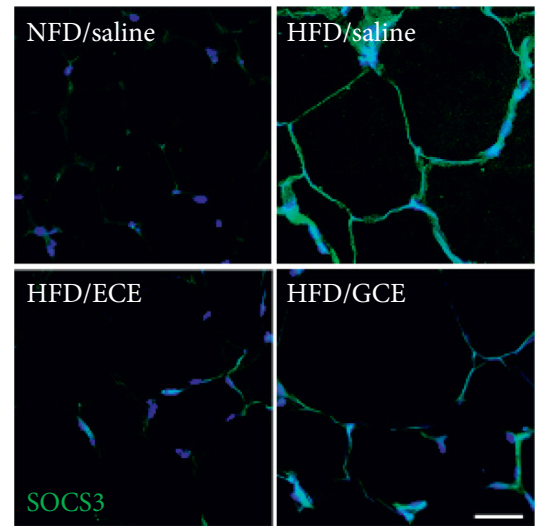

(c)
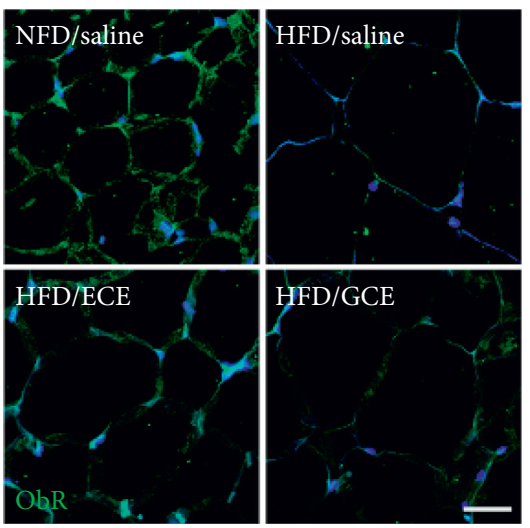

(f)

FIgure 5: Continued. 


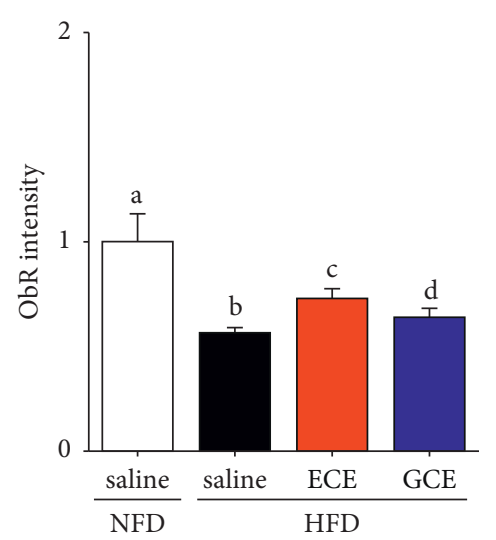

$(\mathrm{g})$

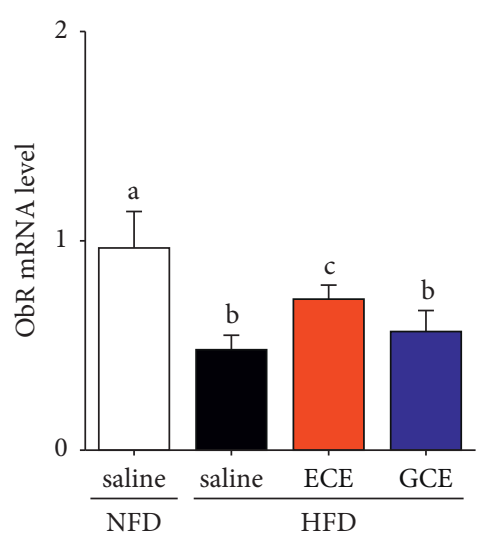

(h)

FiguRE 5: Effects of E. cava extract on serum leptin and adiponectin levels and on leptin resistance in visceral fat of high-fat diet- (HFD-) induced obese mice. (a, b) Serum leptin and adiponectin levels were quantified by ELISA in standard normal fat diet with $0.9 \%$ saline (NFD/ saline; open bar), HFD with $0.9 \%$ saline (HFD/saline; black bar), HFD with E. cava extract (HFD/ECE; red bar), and HFD with Garcinia cambogia extract (HFD/GCE; blue bar) groups. The suppressor of cytokine signaling 3 (SOCS3) as leptin resistance-related molecule protein level (c) (green), (d) quantified intensity graph, and the (e) SOCS3 mRNA expression level was validated by immunostaining and qRT-PCR. Like SOCS3, ObR receptor protein level ((f) green), (g) quantified graph, and (h) the ObR mRNA expression level were validated by immunostaining and qRT-PCR in visceral fat. Nuclei were detected by DAPI (blue) in confocal microscopic images. All mRNA levels expressed relative mRNA level and normalized $B$-actin of the NFD/saline group. Scale bar $=50 \mu \mathrm{m}$. The same letters represent no significant difference $(p<0.05)$.

leptin-stimulated conditions $[51,53]$. Leptin resistance is associated with an early increase in the expression of suppressor of SOCS1 and 3, which inhibits JAK-STAT3 activation [14] and subsequently ObR expression [53]. Similarly, it has been reported that leptin receptor expression is greatly reduced in the adipose tissues of morbidly obese women [54]. We observed that SOCS3 protein and mRNA expression were elevated by HFD but diminished by ECE, which suggests HFD induces leptin resistance in fat tissue by upregulating SOCS3 expression (Figures 5(c)-5(e)). In addition, leptin receptor expression was decreased by HFD and this decrease was increased by ECE. Decreased ObR protein and mRNA expression and localization on adipocyte surfaces are key determinants of cell sensitivity to leptin [15]. Interestingly, PPB also restored leptin sensitivity in HFD-induced obese mice and ob/ob mice [55]. We also found that ECE inhibited HFD-induced decreases in ObR expression, which suggests that ECE enhanced adipocyte leptin sensitivity by maintaining ObR expression (Figures 5(f)$5(\mathrm{~h})$ ).

The HFD-induced condition might be ameliorated by E. cava which plays a direct effect on adipose tissue. The administration of $E$. cava (ECE) suppresses the increasing HFD-induced fat mass and adipocyte size by reducing the food intake, whereas neither the administration of saline nor GCE. Therefore, a possibility can be assured that less mRNA expression of leptin and inflammatory marker in ECE rats might be associated with less fat mass and adipocyte size in ECE compared to the saline and GCE groups.
3.6. Effects of E. cava Extract on Lipolysis in Adipocytes. In vitro, leptin decreases lipogenesis and increases triglyceride hydrolysis and fatty acid oxidation [56, 57]. However, in vivo, the effects of leptin on adipose tissue may be mediated through the peripheral nervous system, and leptin has been reported to increase sympathetic efferent signals to white adipose tissue and thus increase lipolysis $[56,58]$. To initiate lipolysis, the activation of $\mathrm{G} \alpha$ s leads to the activation of adenylyl cyclase (AC), the production of cAMP, which phosphorylates and activates cAMP-dependent protein kinase A (PKA) [59, 60], and the phosphorylation of hormone-sensitive lipase (HSL). Phosphorylated HSL is then translocated from the cytoplasm to the surfaces of lipid droplets and activates the hydrolysis of TG in adipocytes $[61,62]$. Furthermore, it has been suggested that low HSL levels in adipose tissues from obese subjects contribute to the impairment of catecholamine-mediated lipolysis through a postreceptor defect [63]. In our study, the expressions of AC, HSL, and PKA were lower in the HFD/saline group than in the NFD/saline group but significantly higher in the HFD/ECE group than in the HFD/saline group, which suggested that ECE increased adipocyte lipolysis in response to an agonist by leptin in adipocytes (Figure 6).

ECE attenuated leptin resistance by reducing SOCS3 expression and increased leptin sensitivity by increasing leptin receptor expression in adipose tissues, and these modulations increased lipolysis in adipocytes and possibly reduced adipocyte sizes. In addition, it was previously found that the forced expression of SOCS3 increased the expressions of IL-6, TNF- $\alpha$, and MCP-1 but decreased leptin 


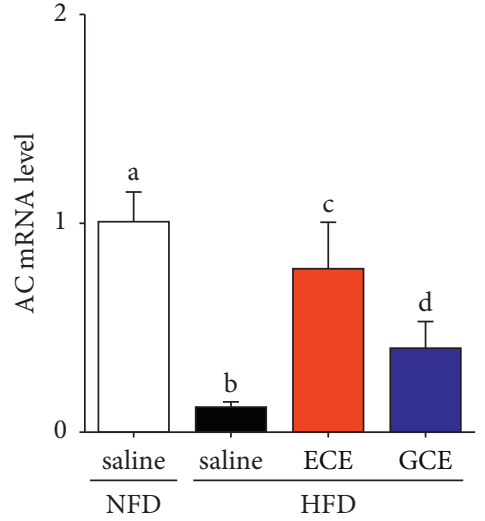

(a)

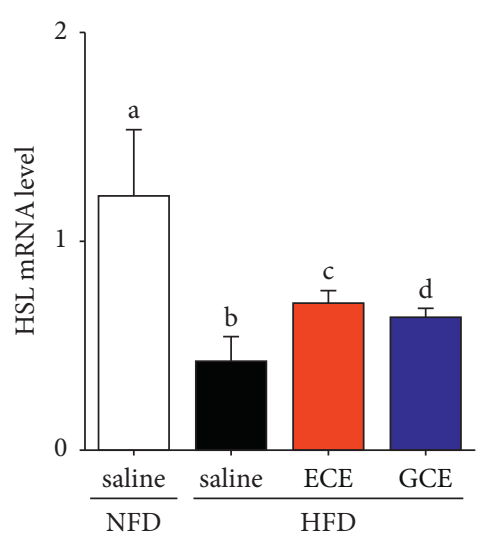

(b)

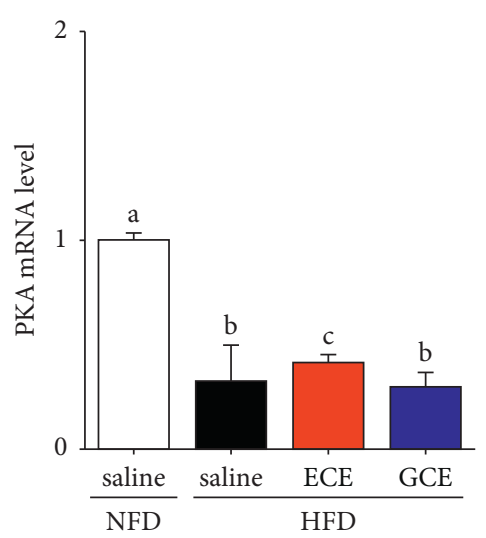

(c)

FiguRE 6: Effects of E. cava extract on adipocyte lipolysis in response to an agonist upregulation in visceral fat of high-fat diet- (HFD-) induced obese mice. To validate adipocyte lipolysis related markers such as (a) AC, (b) HSL, and (c) PKA in visceral fat of standard normal fat diet with $0.9 \%$ saline (NFD/saline; open bar), HFD with $0.9 \%$ saline (HFD/saline; black bar), HFD with E. cava extract (HFD/ECE; red bar), and HFD with Garcinia cambogia extract (HFD/GCE; blue bar) groups, these mRNA expression levels were assessed by qRT-PCR. All mRNA levels expressed relative level and normalized $\beta$-actin of the NFD/saline group. The same letters represent no significant difference $(p<0.05)$.

expressions in 3T3-L1 adipocytes [20]. These observations suggest that SOCS3 promoted 3T3-L1 adipocyte inflammation by promoting the synthesis of inflammatory cytokines and inhibiting leptin signaling [20] and that the ECE induced downregulation of SOCS3 expression might reduce the activities of inflammatory cytokines in adipocytes, which in turn suggests controlling inflammation in fat tissues is important as this inflammation is the main pathophysiology underlying many obesity-related diseases.

In the present study, ECE significantly attenuated body weight, food intake, and adipocyte size increases in HFD-fed mice. Adipocyte hypertrophy creates proinflammatory conditions in fat tissues and leads to macrophage infiltration, M1 polarization, and the activation of CD4 and CD8 T cells, and these immune cells are the primary producers of the proinflammatory cytokines that induce inflammation in fat tissues. ECE administration decreased HFD-induced adiposity and attenuated inflammation in fat tissues by modulating macrophage and T-cell functions and reducing proinflammatory cytokine production and it also attenuated the expressions of TLR4 and RAGE, which might also induce inflammation in adipose tissue. In addition, ECE attenuated leptin resistance in adipose tissue, increased lipolysis, and reduced adipocyte sizes.

\section{Conclusions}

This study shows that E. cava extract (ECE) conferred antiadiposity, anti-inflammatory, and antileptin resistance effects and increased lipolysis in adipocytes. Our results suggest that ECE is a candidate for dietary supplement that inhibits the development of obesity and obesity-associated diseases, especially inflammation-related diseases.

\section{Data Availability}

The data used to support the findings of this study are available from the corresponding author upon request.

\section{Conflicts of Interest}

The authors declare that they have no conflicts of interest.

\section{Authors' Contributions}

Myeongjoo Son and Seyeon Oh contributed equally to this work.

\section{Acknowledgments}

We also thank Aqua Green Technology for providing us with the ECE and GCE used in the study. This research was part of a project entitled "Development of functional food products with natural materials derived from marine resources" (no. 20170285), funded by the Ministry of Oceans and Fisheries, and supported by the Gachon University research fund of 2019 (no. GCU-2019-0330), Republic of Korea.

\section{Supplementary Materials}

Figure S1. High-performance liquid chromatography (HPLC) of E. cava extract. Figure S2. Effects of E. cava extract on NF- $\kappa \mathrm{B}$ expression in visceral fat of high-fat diet(HFD-) induced obese mice. Table S1. List of primers for quantitative polymerase chain reaction (qRT-PCR) and supplementary materials and methods. (Supplementary Materials)

\section{References}

[1] M. Jernås, J. Palming, K. Sjöholm et al., "Separation of human adipocytes by size: hypertrophic fat cells display distinct gene expression," The FASEB Journal, vol. 20, no. 9, pp. 1540-1542, 2006.

[2] S. S. Choe, J. Y. Huh, I. J. Hwang, J. I. Kim, and J. B. Kim, "Adipose tissue remodeling: its role in energy metabolism and metabolic disorders," Frontiers in Endocrinology, vol. 7, Article ID 000300, 2016. 
[3] J. Y. Huh, Y. J. Park, M. Ham, and J. B. Kim, "Crosstalk between adipocytes and immune cells in adipose tissue inflammation and metabolic dysregulation in obesity," Molecules and Cells, vol. 37, no. 5, pp. 365-371, 2014.

[4] M. Kuroda and H. Sakaue, "Adipocyte death and chronic inflammation in obesity," The Journal of Medical Investigation, vol. 64, no. 3, pp. 193-196, 2017.

[5] Y. Masuda, A. Nakanishi, R. Ohashi et al., "Verification of the intermolecular parallel $\beta$-sheet in E22K-A $\beta 42$ aggregates by solid-state NMR using rotational resonance: implications for the supramolecular arrangement of the toxic conformer of A 342 ," Bioscience, Biotechnology, and Biochemistry, vol. 72, no. 8, pp. 2170-2175, 2008.

[6] J. M. Friedman and J. L. Halaas, "Leptin and the regulation of body weight in mammals," Nature, vol. 395, no. 6704, pp. 763-770, 1998.

[7] P. J. Scarpace and Y. Zhang, "Elevated leptin: consequence or cause of obesity?" Frontiers in Bioscience, vol. 1, no. 12, pp. 3531-3544, 2007.

[8] R. Sripradha and S. G. Magadi, "Efficacy of garcinia cambogia on body weight, inflammation and glucose tolerance in high fat fed male wistar rats," Journal of Clinical and Diagnostic Research, vol. 9, no. 2, pp. 1-4, 2015.

[9] C. Vaisse, J. L. Halaas, C. M. Horvath, J. E. Darnell, M. Stoffel, and J. M. Friedman, "Leptin activation of Stat3 in the hypothalamus of wild-type and ob/ob mice but not db/db mice," Nature Genetics, vol. 14, no. 1, pp. 95-97, 1996.

[10] E. Balland and M. A. Cowley, "New insights in leptin resistance mechanisms in mice," Frontiers in Neuroendocrinology, vol. 39, pp. 59-65, 2015.

[11] K. El-Haschimi, D. D. Pierroz, S. M. Hileman, C. Bjørbæk, and J. S. Flier, "Two defects contribute to hypothalamic leptin resistance in mice with diet-induced obesity," Journal of Clinical Investigation, vol. 105, no. 12, pp. 1827-1832, 2000.

[12] A. S. Metlakunta, M. Sahu, and A. Sahu, "Hypothalamic phosphatidylinositol 3-kinase pathway of leptin signaling is impaired during the development of diet-induced obesity in FVB/N mice," Endocrinology, vol. 149, no. 3, pp. 1121-1128, 2008.

[13] M. Jiang, W. Zhang, P. Liu, W. Yu, T. Liu, and J. Yu, "Dysregulation of SOCS-mediated negative feedback of cytokine signaling in carcinogenesis and its significance in cancer treatment," Frontiers in Immunology, vol. 8, 2017.

[14] C. Bjørbaek, K. El-Haschimi, J. D. Frantz et al., "The role of SOCS3 in leptin signaling and leptin resistance," Journal of Biological Chemistry, vol. 274, no. 42, pp. 30059-30065, 1999.

[15] A. Santoro, G. Mattace Raso, and R. Meli, "Drug targeting of leptin resistance,” Life Sciences, vol. 140, pp. 64-74, 2015.

[16] J. A. B. Pedroso, A. M. Ramos-Lobo, and J. Donato, "SOCS3 as a future target to treat metabolic disorders," Hormones, vol. 18, no. 2, pp. 127-136, 2019.

[17] B. Emanuelli, P. Peraldi, C. Filloux et al., "SOCS-3 inhibits insulin signaling and is up-regulated in response to tumor necrosis factor- $\alpha$ in the adipose tissue of obese mice," Journal of Biological Chemistry, vol. 276, no. 51, pp. 47944-47949, 2001.

[18] J. J. Senn, P. J. Klover, I. A. Nowak et al., "Suppressor of cytokine signaling-3 (SOCS-3), a potential mediator of interleukin-6-dependent insulin resistance in hepatocytes," Journal of Biological Chemistry, vol. 278, no. 16, pp. 1374013746, 2003.

[19] K. Ueki, T. Kondo, and C. R. Kahn, "Suppressor of cytokine signaling 1 (SOCS-1) and SOCS-3 cause insulin resistance through inhibition of tyrosine phosphorylation of insulin receptor substrate proteins by discrete mechanisms," Molecular and Cellular Biology, vol. 24, no. 12, pp. 5434-5446, 2004.

[20] Z. Liu, L. Gan, Z. Zhou, W. Jin, and C. Sun, "SOCS3 promotes inflammation and apoptosis via inhibiting JAK2/STAT3 signaling pathway in 3T3-L1 adipocyte," Immunobiology, vol. 220, no. 8, pp. 947-953, 2015.

[21] E. Y. Park, E. H. Kim, M. H. Kim et al., "Polyphenol-rich fraction of Brown alga Ecklonia cava collected from Gijang, Korea, reduces obesity and glucose levels in high-fat dietinduced obese mice," Evidence-Based Complementary and Alternative Medicine, vol. 2012, Article ID 418912, 11 pages, 2012.

[22] W. A. J. P. Wijesinghe and Y.-J. Jeon, "Exploiting biological activities of brown seaweed Ecklonia cava for potential industrial applications: a review," International Journal of Food Sciences and Nutrition, vol. 63, no. 2, pp. 225-235, 2012.

[23] K. A. Kang, K. H. Lee, S. Chae et al., "Triphlorethol-A from Ecklonia cava protects V79-4 lung fibroblast against hydrogen peroxide induced cell damage," Free Radical Research, vol. 39, no. 8, pp. 883-892, 2005.

[24] J. Kang, H. J. Hwang, D. H. Hong et al., "Antioxidant and antiinflammatory activities of ventol, a phlorotannin-rich natural agent derived from Ecklonia cava, and its effect on proteoglycan degradation in cartilage explant culture," Research Communications in Molecular Pathology and Pharmacology, vol. 115-116, pp. 77-95, 2004.

[25] S.-J. Park, G. Ahn, N. H. Lee, J. W. Park, Y.-J. Jeon, and Y. Jee, "Phloroglucinol (PG) purified from Ecklonia cava attenuates radiation-induced apoptosis in blood lymphocytes and splenocytes," Food and Chemical Toxicology, vol. 49, no. 9, pp. 2236-2242, 2011.

[26] I. Wijesekara, N. Y. Yoon, and S.-K. Kim, "Phlorotannins from Ecklonia cava (Phaeophyceae): biological activities and potential health benefits," Biofactors, vol. 36, no. 6, pp. 408-414, 2010.

[27] C. Kang, Y. B. Jin, H. Lee et al., "Brown alga Ecklonia cava attenuates type 1 diabetes by activating AMPK and Akt signaling pathways," Food and Chemical Toxicology, vol. 48, no. 2, pp. 509-516, 2010.

[28] S.-H. Lee, J.-S. Han, S.-J. Heo, J.-Y. Hwang, and Y.-J. Jeon, "Protective effects of dieckol isolated from Ecklonia cava against high glucose-induced oxidative stress in human umbilical vein endothelial cells," Toxicology in Vitro, vol. 24, no. 2, pp. 375-381, 2010.

[29] S. E. Ohia, C. A. Opere, A. M. LeDay, M. Bagchi, D. Bagchi, and S. J. Stohs, "Safety and mechanism of appetite suppression by a novel hydroxycitric acid extract (HCA-SX)," Molecular and Cellular Biochemistry, vol. 238, no. 1-2, pp. 89-103, 2002.

[30] A. C. Sullivan, M. Singh, P. A. Srere, and J. P. Glusker, "Reactivity and inhibitor potential of hydroxycitrate isomers with citrate synthase, citrate lyase, and ATP citrate lyase," Journal of Biological Chemistry, vol. 252, no. 21, pp. 75837590, 1977.

[31] N. H. Roy, M. Lambele, J. Chan, M. Symeonides, and M. Thali, "Ezrin is a component of the HIV-1 virological presynapse and contributes to the inhibition of cell-cell fusion," Journal of Virology, vol. 88, no. 13, pp. 7645-7658, 2014.

[32] J. A. Jablonski, S. A. Amici, L. M. Webb et al., "Novel markers to delineate murine M1 and M2 macrophages," PLoS One, vol. 10, no. 23, Article ID e0145342, 2015.

[33] F. O. Martinez and S. Gordon, "The M1 and M2 paradigm of macrophage activation: time for reassessment," F1000Prime Reports, vol. 6, no. 13, 2014. 
[34] A. Chawla, K. D. Nguyen, and Y. P. S. Goh, "Macrophagemediated inflammation in metabolic disease," Nature Reviews Immunology, vol. 11, no. 11, pp. 738-749, 2011.

[35] B. C. Lee and J. Lee, "Cellular and molecular players in adipose tissue inflammation in the development of obesity-induced insulin resistance," Biochimica et Biophysica Acta, vol. 1842, no. 3, pp. 446-462, 2014.

[36] V. Z. Rocha, E. J. Folco, G. Sukhova et al., "Interferon-gamma, a Th1 cytokine, regulates fat inflammation: a role for adaptive immunity in obesity," Circulation Research, vol. 103, no. 5, pp. 467-476, 2008.

[37] S. Nishimura, I. Manabe, M. Nagasaki et al., "CD8+ effector $\mathrm{T}$ cells contribute to macrophage recruitment and adipose tissue inflammation in obesity," Nature Medicine, vol. 15, no. 8, pp. 914-920, 2009.

[38] S. Winer, Y. Chan, G. Paltser et al., "Normalization of obesityassociated insulin resistance through immunotherapy," $\mathrm{Na}$ ture Medicine, vol. 15, no. 8, pp. 921-929, 2009.

[39] M. Son M, S. Oh, H. S. Lee et al., "Ecklonia cava extract attenuates endothelial cell dysfunction by modulation of inflammation and Brown adipocyte function in perivascular fat tissue," Nutrients, vol. 15, no. 11, p. E2795, 2019.

[40] F. M. Wensveen, S. Valentić, M. Š.lent, T. Turk Wensveen, and B. Polić, "The "Big Bang" in obese fat: events initiating obesity-induced adipose tissue inflammation," European Journal of Immunology, vol. 45, no. 9, pp. 2446-2456, 2015.

[41] J. Mauer, B. Chaurasia, J. Goldau et al., "Signaling by IL-6 promotes alternative activation of macrophages to limit endotoxemia and obesity-associated resistance to insulin," Nature Immunology, vol. 15, no. 5, pp. 423-430, 2014.

[42] D. Wu, A. B. Molofsky, H. E. Liang et al., "Eosinophils sustain adipose alternatively activated macrophages associated with glucose homeostasis," Science, vol. 332, no. 606, pp. 243-247, 2011.

[43] J. H. Hwang, K. J. Kim, and B. Y. Lee, "Crude Ecklonia cava flake extracts attenuate inflammation through the regulation of TLR4 signaling pathway in LPS-induced RAW264.7 cells," Molecules, vol. 22, no. 5, p. E777, 2017.

[44] T. Kawai and S. Akira, "TLR signaling," Cell Death \& Differentiation, vol. 13, no. 5, pp. 816-825, 2006.

[45] R. Donato, "S100: a multigenic family of calcium-modulated proteins of the EF-hand type with intracellular and extracellular functional roles," The International Journal of Biochemistry \& Cell Biology, vol. 33, no. 7, pp. 637-668, 2001.

[46] Y. Yamamoto and H. Yamamoto, "RAGE-mediated inflammation, type 2 diabetes, and diabetic vascular complication," Frontiers in Endocrinology (Lausanne), vol. 4, 2013.

[47] K. H. J. Gaens, G. H. Goossens, P. M. Niessen et al., " $N \mathcal{E}$ (Carboxymethyl) lysine-receptor for advanced glycation end product axis is a key modulator of obesity-induced dysregulation of adipokine expression and insulin resistance," Arteriosclerosis, Thrombosis, and Vascular Biology, vol. 34, no. 6, pp. 1199-1208, 2014.

[48] F. Song, C. Hurtado Del Pozo, R. Rosario et al., "RAGE regulates the metabolic and inflammatory response to high-fat feeding in mice," Diabetes, vol. 63, no. 6, pp. 1948-1965, 2014.

[49] J. Steiner, K. Schiltz, M. Walter et al., "S100B serum levels are closely correlated with body mass index: an important caveat in neuropsychiatric research," Psychoneuroendocrinology, vol. 35, no. 2, pp. 321-324, 2010.

[50] J. Choi, S. Oh, M. Son, and K. Byun, "Pyrogallol-Phloroglucinol6,6-Bieckol alleviates obesity and systemic inflammation in a mouse model by reducing expression of RAGE and RAGE ligands," Marine Drugs, vol. 17, no. 11, p. E612, 2019.
[51] R. B. Harris, "Direct and indirect effects of leptin on adipocyte metabolism," Biochimica et Biophysica Acta (BBA)-Molecular Basis of Disease, vol. 1842, no. 3, pp. 414-423, 2014.

[52] Z. Wang, Y.-T. Zhou, T. Kakuma et al., "Leptin resistance of adipocytes in obesity: role of suppressors of cytokine signaling," Biochemical and Biophysical Research Communications, vol. 277, no. 1, pp. 20-26, 2000.

[53] M.-Y. Wang, L. Orci, M. Ravazzola, and R. H. Unger, "Fat storage in adipocytes requires inactivation of leptin's paracrine activity: implications for treatment of human obesity," Proceedings of the National Academy of Sciences, vol. 102, no. 50, pp. 18011-18016, 2005.

[54] K. Séron, L. Corset, F. Vasseur et al., "Distinct impaired regulation of SOCS3 and long and short isoforms of the leptin receptor in visceral and subcutaneous fat of lean and obese women," Biochemical and Biophysical Research Communications, vol. 348, no. 4, pp. 1232-1238, 2006.

[55] M. Son, S. Oh, J. Choi et al., "Attenuation of inflammation and leptin resistance by pyrogallol-phloroglucinol-6,6-bieckol on in the brain of obese animal models," Nutrients, vol. 14, no. 11, p. E2773, 2019.

[56] J. H. Stern, J. M. Rutkowski, and P. E. Scherer, "Adiponectin, leptin, and fatty acids in the maintenance of metabolic homeostasis through adipose tissue crosstalk," Cell Metabolism, vol. 23, no. 5, pp. 770-784, 2016.

[57] W. N. William Jr., R. B. Ceddia, and R. Curi, "Leptin controls the fate of fatty acids in isolated rat white adipocytes," Journal of Endocrinology, vol. 175, no. 3, pp. 735-744, 2002.

[58] T. J. Bartness, C. K. Song, H. Shi, R. R. Bowers, and M. T. Foster, "Brain-adipose tissue cross talk," Proceedings of the Nutrition Society, vol. 64, no. 1, pp. 53-64, 2005.

[59] R. C. Honnor, G. S. Dhillon, and C. Londos, "cAMP-dependent protein kinase and lipolysis in rat adipocytes II. definition of steady-state relationship with lipolytic and antilipolytic modulators," Journal of Biological Chemistry, vol. 260, no. 28, pp. 15130-15138, 1985.

[60] A. S. Greenberg, W.-J. Shen, K. Muliro et al., "Stimulation of lipolysis and hormone-sensitive lipase via the extracellular signal-regulated kinase pathway," Journal of Biological Chemistry, vol. 276, no. 48, pp. 45456-45461, 2001.

[61] C. Holm, "Molecular mechanisms regulating hormone-sensitive lipase and lipolysis," Biochemical Society Transactions, vol. 31, no. 6, pp. 1120-1124, 2003.

[62] J. Ogasawara, T. Izawa, T. Sakurai et al., "The molecular mechanism underlying continuous exercise training-induced adaptive changes of lipolysis in white adipose cells," Journal of Obesity, vol. 2015, Article ID 473430, 10 pages, 2015.

[63] J. J. Egan, A. S. Greenberg, M. K. Chang, S. A. Wek, M. C. Moos, and C. Londos, "Mechanism of hormonestimulated lipolysis in adipocytes: translocation of hormonesensitive lipase to the lipid storage droplet," Proceedings of the National Academy of Sciences, vol. 89, no. 18, pp. 8537-8541, 1992. 